sie aber im qualifikationsgebundenen $\mathrm{Zu}$ satzvolumen (QZV) enthalten ist, wird sie mit $37,31 €$ wie der Pricktest bezahlt. PKV und BG vergüten die spezifische Provokation mit 344,10 bzw. 250,92 €. „Man sollte aber tun, was sinnvoll ist", betonte Lepp, „und nicht, was bezahlt wird“.

\section{Orale Provokation:}

für die Praxis zu riskant

Aufgrund der Gefahr einer systemischen anaphylaktischen Reaktion werden orale Provokationen fast immer stationär durchgeführt. Wie Dr. Laura Maintz, Universitätsklinikum Bonn, erklärte, beträgt das Basisentgelt 1.139,77 € bei einer Liegedauer von zwei bis fünf Tagen.
Dieses errechnet sich aus dem (landesabhängigen) Basisfallpreis (2.991,53€) und dem Basiskostengewicht $(0,381)$.

Für die Arzneimittelprovokation liegt der optimale Zeitpunkt zwischen vier Wochen und sechs Monaten nach der Reaktion. Wenn die Reaktion jedoch schon jahrelang zurückliegt, besteht die Gefahr, dass eine vorübergehend verlorengegangene Überempfindlichkeit durch die erneute Exposition wieder geboostert wird. Bei Verdacht auf eine Reaktion durch die Kombination von Auslösern sollte eine Co-Exposition gegenüber dem Co-Stimulus erfolgen, also zum Beispiel bei Verdacht auf eine Anstrengungsanaphylaxie erst die Medikamentengabe, dann das
Laufbandtraining. Falsch-negative Ergebnisse können auch durch zu niedrige Provokationsdosen bedingt sein, ebenso durch eine Toleranzentwicklung bei Testung mit ansteigenden Dosen. Speziell bei Verdacht auf Histaminunverträglichkeit riet Maintz grundsätzlich zur Provokation, auch wenn dies nur von wenigen Zentren durchgeführt werde. Die Bestimmung der DAO-(Diaminoxidase-)Aktivität sei weder sehr sensitiv noch spezifisch und werde auch in den aktuellen Leitlinien nicht mehr empfohlen. Dr. Elke Oberhofer

Fortbildungssymposium „Provokationen kritisch gesehen: Was ist validiert? Was machbar? Was wird bezahlt?"

\title{
Therapieallergeneverordnung: mehr Qualität, aber weniger Vielfalt
}

\section{Seit 2008 gilt in Deutschland die Therapieallergeneverordnung. Derzeit laufen noch Übergangsfristen von mindestens acht Jahren - also bis 2018 und länger. Beim Allergiekongress war den Auswirkungen auf die Praxis des niedergelassenen Allergologen ein eigenes Seminar gewidmet.}

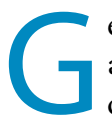
eschaffen wurde die Therapieallergeneverordnung (TAV) mit dem Ziel, die zahlreichen Präparate, die für die spezifische Immuntherapie (SIT) häufiger Allergien zur Verfügung stehen, hinsichtlich deren Qualität, Wirksamkeit und Sicherheit zu überprüfen. Individualrezepturen sollen zukünftig nur für seltenere Allergenquellen eingesetzt werden. Die TAV gilt für Süßgräser, Birke, Erle, Hasel, Hausstaubmilben sowie Bienen- und Wespengift. Derzeit befinden sich 101 Produkte im Zulassungsverfahren beim Paul-Ehrlich-Institut (PEI), wie Prof. Dr. Stefan Vieths, Vizepräsident des PEI berichtete. In den letzten zwei Jahren wurden 19 Neuanträge auf klinische Prüfung gestellt. Die größten Probleme, auf die das PEI bei den bisherigen Anträgen gestoßen ist, sind unzureichende Konzepte bei den Dosisfindungsstudien sowie mangelhafte Qualitätsdaten der Allergenprodukte. Vieths fast die derzeitige Situation folgendermaßen zusammen: „Einerseits wird sich die Wirksamkeit und Sicherheit der Allergenprodukte, die zur Behandlung häufiger Allergien auf dem Markt verbleiben, er- heblich verbessern, andererseits wird sich die Therapievielfalt verringern."

Was hat die Umsetzung der TAV für Auswirkungen auf die Therapiemöglichkeiten im ärztlichen Alltag? Diese Frage beleuchtete PD Dr. Jörg Kleine-Tebbe, Berlin, am Beispiel der Frühblüherallergie. Bei Patienten, die auf Haselnuss-, Erlen- und Birkenpollen reagieren, wird sich durch die TAV nichts ändern. Aufgrund der großen Verwandtschaft zwischen den drei Allergenen ist es ohnehin ausreichend, die SIT z.B. nur mit Birke durchzuführen. Die Allergene der drei Bäume gehören alle zur TAV und müssen daher das Zulassungsverfahren durchlaufen. Es gibt hier schon jetzt viele Präparate auf dem Markt und die wird es nach Einschätzung von KleineTebbe auch künftig geben. Was aber, wenn noch zwei weitere Bäume wie Buche und Eiche hinzukommen? Auch hier stellt sich aufgrund der Verwandtschaft mit dem Hauptallergen der Birke die Frage, ob man solche Bäume überhaupt berücksichtigen muss. Möchte man sie jedoch explizit in die Therapie mit einbeziehen, so kann man dies ab 2018 nur noch getrennt als Indivi- dualrezeptur, nicht als Mischung mit Haselnuss, Erle und Birke. Hintergrund: Buche und Eiche sind nicht Bestandteil der TAV. Auch Patienten, die beispielsweise allergisch auf Bäume, Gräser und Alternaria reagieren, müssen jeweils mit 100\%igen Extrakten einzeln behandelt werden, eine Mischung ist auch hier ab 2018 nicht zulässig. Auch Kombinationen aus beispielsweise Baum- und Gräserpollenallergenen dürfen ohne Zulassungsverfahren nicht mehr genutzt werden, sondern müssten ab 2018 einzeln zum Einsatz kommen.

Insgesamt ist Kleine-Tebbe für die $\mathrm{Zu}$ kunft zuversichtlich: „Auch nach 2018 werden wir mit Hilfe der wichtigen Therapieallergenkombinationen und den Individualrezepturen - wenn auch nicht gemischt mit den TAV-Allergenen durchaus die Möglichkeit haben, individuell zu therapieren." Marion Weber

Interaktives Seminar „Folgen der Therapieallergeneverordnung"

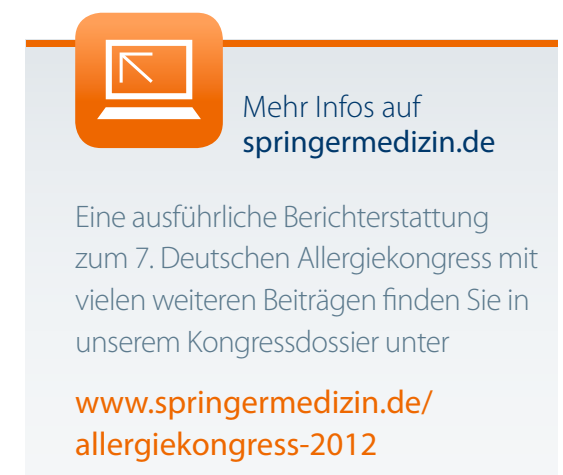

